\title{
Pain Management in Children During the COVID-19 Pandemic
}

\author{
Patricia A. Richardson ${ }^{1,2}$ (D) Anjana Kundu ${ }^{3,4}$ \\ Accepted: 28 June 2021 / Published online: 26 July 2021 \\ (C) Springer Science+Business Media, LLC, part of Springer Nature 2021
}

\begin{abstract}
Purpose of Review For many children, the SARS-CoV-2 pandemic has impacted the experience and treatment of their pain. This narrative review draws from the pain literature and emerging findings from COVID-19 research to highlight potentially meaningful directions for clinical consideration and empirical inquiry in the months and years to come.

Recent Findings COVID-19 has been linked to diffuse acute pains as well as chronic pain sequelae. Contextual factors known to increase vulnerability for pain and associated functional disability have been exacerbated during the pandemic. Beyond these salient concerns has been the remarkable resilience demonstrated by patients and providers as healthcare systems have sought to harness creativity and innovative digital solutions to support optimal child wellbeing throughout this crisis.

Summary Ongoing research is needed to elucidate the short- and long-term effects of the pandemic on children's pain and to consider how the delivery of treatment via digital technology has impacted existing paradigms of pain management.
\end{abstract}

Keywords COVID-19 $\cdot$ Pediatric $\cdot$ Pain $\cdot$ Acute pain $\cdot$ Chronic pain $\cdot$ MIS-C $\cdot$ Telehealth $\cdot$ Sleep $\cdot$ Abuse $\cdot$ Neglect $\cdot$ Virtual care . School $\cdot$ Psychosocial $\cdot$ Behavioral

\section{Introduction}

There have been over 27 million documented cases of severe acute respiratory syndrome coronavirus 2 (SARS-CoV-2) in the USA and over 108 million cases worldwide. Although the impact of the coronavirus disease 2019 (COVID-19) pandemic has been felt by all, there are special considerations to make for children experiencing pain. The pandemic has had a profound effect on the way that pain is experienced by children and families. Not only are headache and diffuse pains

This article is part of the Topical Collection on Pediatric Anesthesia

Patricia A. Richardson

panri@msu.edu

1 Departments of Pediatric Psychology and Pediatric Pain and Palliative Medicine, Helen DeVos Children's Hospital, 35 Michigan St. NE., Grand Rapids, MI 49503, USA

2 Department of Pediatrics and Human Development, Michigan State University College of Human Medicine, East Lansing, MI, USA

3 Department of Pediatric Anesthesiology, Dayton Children's Hospital, Dayton, OH, USA

4 Department of Pediatrics, Wright State University Boonshoft School of Medicine, Dayton, OH, USA symptoms of the virus [1•], but a unique presentation among older children, multisystem inflammatory syndrome (MIS-C), has manifested as severe abdominal pain, joint swelling, and pain [2]. Furthermore, contextual factors known to increase vulnerability for pain and associated functional disability have been higher within the context of the pandemic (e.g., stress, anxiety, depression, social isolation, sleep disturbance, reduced activities of daily living, physical deconditioning) $[3 \cdot 4,5]$.

COVID-19 has also impacted optimal pain management for children due to safety and logistical challenges. Depending on location of residence, there were weeks to months at the outset of the pandemic when healthcare professionals, inpatient and outpatient, were unable to see patients. During this period, healthcare systems rapidly developed safety protocols and digital technology infrastructure to support virtual visits. Across many healthcare systems, restrictions were placed on elective, routine, and nonemergency interventional procedures, postponing them unless considered essential. Some pain professionals were redistributed from primary domains of practice to assist with the urgent need to evaluate and treat patients with COVID-19. Given that many emergency departments have gone through periods of being inundated with COVID-19 patients, some children with significant pain were either unable to access emergency care or faced delays in 
receiving care. Some families likely elected to not seek painrelated care or to delay visits with the goal of mitigating COVID-19 exposure risk. Some families were likely challenged by the quality and reliability of virtual visits, or lacked the tech acumen to access virtual visits [6,7]. Adding complexity to all of the above is that rates of COVID-19 across geographic areas fluctuated over time, with resultant ongoing variabilities in access to care.

We are only in the beginning stages of understanding the impact of the COVID-19 pandemic on pain management for children and families, though indications are that it may have a deleterious effect $[8 \cdot \bullet, 9]$. Despite the risk and challenges, the medical community has demonstrated resilience in rapidly mobilizing to meet the demands of these unprecedented and evolving circumstances and ensure that children safely receive the care they need. The aim of this narrative review is to highlight meaningful directions for clinical consideration and empirical inquiry in the months and years to come. There is limited available data specific to pediatric pain during the pandemic. Thus, this review draws from extant pain research and COVID-19-specific work outside of the pain literature to shed light on potentially meaningful domains for children with pain.

Literature for this narrative review was identified through a non-systematic search in PubMed and Google Scholar using the following search terms: COVID-19, child, pediatric, adolescent, pain, acute pain, chronic pain, psychosocial, MIS-C, sleep, physical activity. Studies were screened for inclusion by title and abstract. No language or other restrictions were applied. We also searched reference lists of papers that were identified as particularly relevant and reviewed studies with foundational or innovative approaches to key domains of inquiry.

\section{Impact of COVID-19}

\section{Pain Overview}

Pain is a multidimensional biopsychosocial process, where one's experience of pain is related to interacting biological, cognitive, behavioral (e.g., health behaviors), and sociocultural influences [10,11,12,13]. Broadly, pain is conceptualized based on duration - either acute or chronic. Acute pain often occurs within the context of accidents and injuries or as part of routine, illness, or postsurgical care and is expected to resolve when the underlying cause of pain is addressed. Treatment, when needed, may include pharmacological interventions, regional anesthesia, and self-management skills, and other therapies (e.g., physical therapy) as modalities [14]. Chronic pain (CP) describes any pain lasting longer than 3 months. Between 11 and $38 \%$ of children worldwide experience CP [15]. Persistent pain has adverse effects on children's social, emotional, and academic function [16,17,18]. Per the ICD-11 and guidelines from IASP, CP can be understood either as pain that is the disease itself (chronic primary pain) or pain that develops due to another disease (chronic secondary pain). Optimal evidence-based treatment for $\mathrm{CP}$ involves a functional rehabilitation approach, with interdisciplinary providers including behavioral health, physical therapy, and pain medicine among others (e.g., complementary and integrative approaches, occupational therapy) [19]. These services may be provided in inpatient or outpatient settings depending on presentation and degree of functional decline at intake.

The effect of the COVID-19 pandemic on children's pain will be discussed across the following domains [1]: the direct impact of COVID-19 on pain [2], psychosocial and behavioral considerations, and [3] impacts of COVID-19 on pain interventions.

\section{Impact of COVID-19 on Pain}

There are several risk factors that predispose children to experience pain during or as a consequence of infection with SARS-CoV-2 in the acute setting or as a long-term consequence $[8 \cdot \bullet, 20 \bullet \bullet$. Those with pre-existing pain diagnoses may be at a greater risk for worsening of their pain and/or development of new pain symptoms.

Despite their relatively lower risk of contracting COVID19 as compared to adults, children with COVID-19 may experience fatigue, myalgias, abdominal pain, chest pain, and headaches with or without respiratory symptoms. Some children with SARS-CoV-2 develop MIS-C, a concerning condition most common among older children that disproportionately impacts children of color (African American and Hispanic) $[21,22,23 \cdot \bullet]$. The alarming symptoms of fever, hypotension, cardiac dysfunction, severe abdominal pain, headache, skin rash, and inflammation of the joints and mucosa often make it clinically indistinguishable from acute abdominal emergencies or Kawasaki disease. Common symptoms of MIS-C are abdominal pain (up to $71 \%$ ), headache, and joint pain, but the long-term sequelae remain unknown. The pathophysiology of MIS-C is hypothesized to be a delayed inflammatory response secondary to inappropriate stimulation of the innate immune system, and significant hyperinflammation and cytokine release $[21,22,23 \cdot \bullet, 24,25]$. Considering the role of overproduction of inflammatory mediators in chronic pain, MIS-C represents a simulated risk for the development or worsening of chronic pain or an autoimmune condition.

Literature clearly supports viral infections as triggers in the onset of acute and persistent pain for some patients as seen after influenza, H1N1, and SARS coronavirus [26,27]. Chronic post-SARS syndrome, with symptoms including fatigue, diffuse myalgia, depression, and nonrestorative sleep lasting almost 2 years, has been documented [28]. Similarly, specific postinfectious syndrome of pain, fatigue, and memory 
difficulties for up to 12 months may ensue after infection with the Ross River virus (epidemic polyarthritis), Coxiella burnetii (Q fever), and Epstein-Barr virus (infectious mononucleosis) [29]. Postinfectious irritable bowel syndrome has been found to develop secondary to an acute viral or bacterial gastroenteritis among $10 \%$ of patients; premorbid psychosocial vulnerability is a known risk factor [30].

An additional consideration within the context of COVID19 is related to hospital admission. From the broader literature, we know that prolonged intensive care unit (ICU) admissions increase risk of developing long-lasting severe functional limitations, psychological distress, and CP [31-33]. Thus, these outcomes should be closely attended to for children hospitalized at length with COVID-19.

\section{Psychosocial and Behavioral Considerations}

\section{Stress and Mental Health}

Psychosocial and behavioral factors have been found to "predispose people to experience pain, be a precipitant of symptoms, be a modulating factor amplifying or inhibiting the severity of pain, be a consequence of persistent pain, or be a perpetuating factor." [13-15] The pain literature is replete with evidence identifying interrelations among psychosocial factors and pain. For example, preprocedural anxiety has been found to increase children's experience of discomfort during routine medical procedures, such as venipuncture [34]. Presurgical anxiety and pain coping efficacy have been found to predict which patients continue to experience pain at 12 months post-operation [35]. Anxiety, depression, and painspecific anxiety have all been associated with the development and continuity of chronic pain among children [36-39]. Thus, it would be expected that COVID-19-related stress would lend itself to increased psychosocial distress among children and families. A preliminary literature search has found that children's anxiety and depressive symptoms have been higher since the onset of the pandemic [40]. In one survey study of youth in the UK between the ages of 13 and 25 years with a history of mental health concerns, findings indicated that up to $51 \%$ of the sample believed that their mental health deteriorated and $26 \%$ reported difficulty accessing mental health treatment during the pandemic [41]. It will be important to evaluate psychosocial risk within pediatric pain samples as these data become available.

\section{School}

It is well-documented in the literature that experiencing $\mathrm{CP}$ has a significant effect on children's school functioning. Youth with CP have increased school absences, schoolrelated anxiety, and declines in academic performance associated with pain $[42,43]$. Children with CP may also have fewer friends, be more socially isolated, and more likely to experience bullying compared to youth without pain [44]. Achieving consistent school attendance and increased engagement with schoolwork is frequently a key goal of functional rehabilitation for children with pain. COVID-19 resulted in school closures and varied periods of virtual or hybrid learning (in-person and virtual) for many youth. This instability in the school environment is expected to result in learning losses for children [45]. Beyond acquisition of academic knowledge, there are multiple ways that school instability has likely impacted youth, including reduced daily structure, peer support, opportunities for physical activity, access to appropriate nutrition (up to $14 \%$ of American children face food insecurity), mental health treatment and other therapies, along with many meaningful supports not included here [46]. School districts have worked hard to overcome these challenges. However, given the school-related vulnerabilities for children with pain, it is possible that children with pain might be disproportionately impacted by inconsistencies in the school environment. This could be exacerbated further for youth with CP who also experience mental health, medical, and learning comorbidities (e.g., ADHD, learning disabilities) and environmental challenges, such as families facing poverty and socioeconomic decline secondary to the COVID-19 economic downturn.

\section{Physical Activity and Routines}

Interdisciplinary interventions for $\mathrm{CP}$ often include physical and occupational therapies, as insufficient daily activity is related to worse pain-related outcomes $[47,48]$. It is common for children with persistent pain to withdraw from extracurricular activities and athletics and have more difficulty maintaining daily routines that support health and wellbeing (e.g., school attendance, nutrition, and sleep schedules). Quarantines, shut-downs, and physical distancing measures have impacted the level of activity and daily structure children can maintain. For example, virtual or hybrid learning at school has facilitated continuity of education to some degree, but simultaneously, significantly increased children's daily screen time with less opportunity for physical activity, maintaining routines, and social engagement. Self-report data collected during COVID-19 with an adult sample of individuals with $\mathrm{CP}$ found that decreases in levels of physical exercise were associated with increased pain [49]. These associations will be important to examine in pediatrics.

\section{Sleep}

Recognizing the bidirectional relationship between sleep and pain has been instrumental in addressing pain intensity as well the efficacy of treatment outcomes [50]. With the COVID-19 pandemic, there is a heightened risk for the onset of sleep 
disturbances. Adolescents with preexisting psychopathologies (including anxiety and depression) and neurodevelopmental conditions (attention-deficit/hyperactivity disorder and autism spectrum disorder) may be especially vulnerable to disturbed sleep during this period [51]. Additionally, social distancing measures, social isolation, and altered school schedule with an increased exposure to digital screens and reduced physical activity all further the risk of sleep disturbances.

\section{Child Abuse and Neglect}

Trauma and adverse experiences in childhood (ACES: physical, mental, or sexual abuse, emotional or physical neglect, a violent home environment, household substance abuse, exposure to parent mental illness, parental separation or divorce, and parental incarceration) are related to $\mathrm{CP}$ and pain-related disability for children and adults [52 •,53,54,55•]. Developmental research has found that abuse is most commonly perpetrated by primary caregivers and is more likely to occur in familial environments characterized by stress, social isolation, substance use, and when children have special needs (e.g., chronic illness) [56]. The multidimensional economic and psychosocial stressors generated by the COVID-19 pandemic (e.g., job loss, economic instability, reduced social interaction) seem to align with environmental risk factors for abuse. It is therefore concerning that children have had even less face-to-face access to mandated child abuse reporters at this time, including mental health and medical providers, teachers, and childcare providers [57•,58,59]. Attending to children's safety, as well as caregiver and familial wellbeing, is always warranted, but may be particularly important in the wake of COVID-19.

\section{Impact of COVID-19 on Pain Interventions}

Even prior to the COVID-19 pandemic, pediatric pain has been underrecognized and undertreated pediatrics $[60,61]$. This may be attributable to historical challenges with assessing pain in young children, shortage of services outside of university and urban centers, significant treatment-related costs, and long provider waitlists, among others [61-65]. Undertreating pain has consequences. Poorly managed acute pain in childhood is a risk for progression to chronic pain in childhood, which in turn leads to a higher risk for CP in adulthood. Thus, timely, efficient, and effective interventions are necessary to interrupt risk for pain and associated functional disability across the lifespan. The pandemic has further highlighted access barriers to appropriate and timely pain care, particularly as it relates to in-person visits with the healthcare professionals.

\section{Triaging and Education}

A careful and intentional triaging system that evaluates the acuity of children's pain care needs (cancer, sickle cell crisis, CRPS) would allow timely treatment access balanced with risk mitigation strategies. For example, it would be beneficial for triaging to indicate when it is appropriate for children to receive virtual versus in-person treatment. It will also likely be important to increase pain education for healthcare professionals, patients, and caregivers to include risk factors for developing and/or worsening pain related to COVID-19, how to minimize that risk, impact of contextual factors (stress, physical activity, sleep), and enhancing self-management strategies. Multimodal and interdisciplinary treatments must be prioritized when possible.

\section{Virtual Care}

A primary shift in the field secondary to COVID-19 has been the rapid adoption of virtually delivered care. Over the past year, virtually delivered care (i.e., telehealth) transitioned from being a promising service delivery method to an essential medium for providers to interface with patients $[66,67 \cdot]$. The time between initial shut-down orders and telehealth mobilization varied greatly across medical systems, with those already employing telehealth having the benefit of existing infrastructure to build from. Telehealth has enabled the pediatric pain management community to increase staff, patient, and family safety, while continuing to provide needed interventions during the pandemic. Beyond telehealth, COVID-19 has resulted in a proliferation of digital health tools in pain management and healthcare. Providers have harnessed creativity and innovation to connect with patients through mobile applications, social media, blogs, podcasts, and online support groups, to name a few (Table 1). The rise of digital health technology in pain management has the potential help to improve access to evidence-based care for more children and address treatment barriers the field has struggled with for decades.

Although telehealth and digital health technology has many strengths and promising directions for the future of pain management, it has also exposed inconsistencies and vulnerabilities of our healthcare system in leveraging technology. Issues at the forefront of technology in healthcare include its accessibility, affordability, safety, and reliability as a viable alternative to in-person care, with varying policies governing its use in healthcare $[20 \bullet \bullet, 67 \bullet]$. Telehealth cannot yet replace in-person physical examination for the accurate assessment of some pain conditions, nor can it replicate the strengths of some treatment modalities (e.g., massage, acupuncture, and aqua therapy). Even when institutions establish well-running, feasible telehealth platforms, it is still necessary for patients to have access to at-home technology to support virtual visits and 
Table 1 Pediatric pain educational and self-management resources

\begin{tabular}{|c|c|c|}
\hline Resource & Description & Who it is for \\
\hline \multicolumn{3}{|l|}{ Organizations/Societies } \\
\hline $\begin{array}{l}\text { International Association for Study of Pain Special } \\
\text { Interest Group on Pain in Childhood (IASP Child } \\
\text { Pain) } \\
\text { http://childpain.org/ }\end{array}$ & $\begin{array}{l}\text { An international multidisciplinary organization focused } \\
\text { specifically on pain research and treatment. } \\
\text { It hosts the International Symposium on Pediatric Pain (ISPP) } \\
\text { interdisciplinary pediatric pain conference. }\end{array}$ & Healthcare Professionals \\
\hline $\begin{array}{l}\text { Society for Pediatric Pain Medicine (SPPM) } \\
\text { https://www.pedspainmedicine.org/ }\end{array}$ & $\begin{array}{l}\text { Multidisciplinary, interprofessional organization driving } \\
\text { excellence in pediatric pain care through education, research, } \\
\text { quality assessment and improvement, development of standards } \\
\text { for pediatric medicine practice and through advocacy. It hosts an } \\
\text { annual conference on pediatric pain. }\end{array}$ & $\begin{array}{l}\text { Healthcare Professionals, } \\
\text { Patients/Families }\end{array}$ \\
\hline
\end{tabular}

Online resources

The Comfort Ability Program https://www.thecomfortability.com/

\section{ImagineAction http://imaginaction.stanford.edu/ Solutions for Kids in Pain \\ (SKIP) https://www.kidsinpain.ca/}

\section{ACI Pain Management Network PainBytes http://www.aci.health.nsw.gov. au/chronic-pain/painbytes \\ Project ECHO (Extensions for Community Healthcare Outcomes) https://hsc.unm.edu/echo/about-us/}

SickKids Online Pediatric Pain Curriculum (OPPC) https://www.sickkids.

ca/en/care-services/centres/pain-centre/\#oppc

Proactive Pain Solutions

https://www.proactivepainsolutions.com/

\section{Apps and m-Health \\ WebMAP Android Users \\ iOS users}

Pain Squad
https://apps.apple.
com/us/app/pain-squad/id929781246
Migraine Buddy Android iOS
Stop, Think \& Breathe
https://apps.apple.
com/ca/app/stop-breathe-think-kids/id1215758068
Calm https://www.calm.com/
Headspace Android iOS

Insight Timer Android iOS

Support groups

Creative Healing for Youth in Pain (CHYP) https://mychyp.org/

The Coalition Against Pediatric Pain (TCAPP) https://tcapp.org/

Pediatric Pain Warriors (US Pain Foundation) https://pediatricpainwarrior.org/
Rooted in pain psychology, this program provides kids, teens and parents/caregivers education and self management tools to better manage chronic pain, post-surgical pain, or any other kind of ongoing pain. It also gives kids and parents the chance to learn and practice many proven coping skills based in cognitive behavioral therapy.

The Comfort Ability Program offers workshops, health chats, newsletters, and other resources for teens and parents.

Self hypnosis program to help with pain, anxiety, stress, mood disturbances.

Evidence-based pediatric pain management knowledge mobilization network, that seeks to bridge the gap between current treatment practices and available evidence-based solutions for children's pain in Canadian health institutions.

Educational videos about chronic pain, self management strategies and treatment options

ECHO connects Health care providers with an inter-professional specialist team through periodic videoconferencing sessions. The sessions include didactic lectures and interactive discussion of de-identified patient cases. Healthcare providers who attend ECHO gain enhanced skills and confidence in managing complex chronic conditions safely and effectively

Online education platform for health-care professionals to learn about pain with clinical, basic science and ethical themes.

Online education and consultation platform in pediatric pain patients and families

App providing pain education, stress \&, emotions management, relaxation, lifestyle and school interventions; staying active; and maintenance and relapse prevention

An app to track and measure pediatric pain,

Patients(10-17 years) \& Families

Children and Adolescents

Patients/Families

Healthcare Professionals

Youth, Families

Healthcare Professionals

Healthcare Professionals

Healthcare Professionals

Healthcare Professionals

Patients/Families

Children (10-18yrs),

Parents/Caregivers

Children (8-18yrs)

Daily symptom tracking app for migraine and headache. Includes free pain tracking and educational tools.

Short, free mindfulness, guided meditation sessions geared towards kids

Guided meditation app for older teens and adults, sleep stories for younger kids

Guided meditations with some content geared towards kids and with five themes: Calm, Focus, Kindness, Sleep, and Wake Up.

Meditation and mindfulness app with pediatric specific content to help with management of anxiety, stress and for relaxation.

A nonprofit online resource for teens with chronic pain and their parents, providing educational resources, exposure to creative healing experiences and social support.

Non-profit committed to improving quality of life for children living with chronic pain from rare diseases through support for children \& families, increasing awareness, funding research, pediatric pain resources for families and medical professionals

Provides resources, education, support, and retreats for children affected by pediatric pain and their families
Children, Adults

Children

Children, Adults/Caregivers

Children, Adults/Caregivers

Children, Adults/Caregivers

Teens, Parents/Caregivers

Children, Families/Caregivers

Healthcare Professionals

Children, Families/Caregivers 
Table 1 (continued)

\begin{tabular}{lll}
\hline Resource & Description & Who it is for \\
\hline $\begin{array}{l}\text { Podcasts } \\
\text { Pedia Pain Focus }\end{array}$ & $\begin{array}{l}\text { Weekly podcast discussing all things related to improving pediatric } \\
\text { pain care, providing education and resources for healthcare } \\
\text { professionals. }\end{array}$ & Heare Professionals \\
\hline
\end{tabular}

to have some degree of comfort and skill in using the technology. There is a "digital divide" related to age and to socioeconomic status. Access to high-speed broadband is lacking for $39 \%$ of rural Americans, and when available, it is often more expensive and slower [6,7]. With increased demand during COVID-19, telecommunication systems have been stressed, further slowing upload and download speeds below usual standards. These factors directly impact the quality and reliability of connectivity for virtual visits. It is imperative that ongoing research address the issues of accessibility, reliability, safety, compliance, affordability, care coordination, regulation, and reimbursement for services provided using digital technology.

Beyond the necessity of adequate access to and comfort with technology, there are other aspects of telehealth in pain management that need to be better understood. For example, it is unknown the degree to which telehealth could impact interdisciplinary care and care coordination among pain providers. If interdisciplinary communication is undermined, and treatment is perceived by patients as fragmented, there could be impacts on quality of care, patient engagement, and patient satisfaction. It is also possible that an unintended lack of care coordination could lead to an increased reliance on biomedical management (e.g., medications including opioids), rather than the multimodal treatment including psychological and rehabilitation approaches [67•].

\section{Medical Management}

Given increased barriers to treatment access secondary to COVID-19, the relaxed regulatory measures around controlled medications may have inadvertently promoted reliance on medications as opposed to interdisciplinary treatment [68]. From pre-COVID-19 research, we know that youth most commonly access illicit opioids via prescriptions of family members and misuse opioids with the goal of pain management [69•]. Pain professionals must make every attempt to ensure provision of timely and coordinated interdisciplinary pain care when possible, while ensuring continued education and surveillance measures around medication prescription and safety.

\section{Provider Wellbeing}

It is important to consider possible implications of the COVID-19 pandemic on the wellbeing of interdisciplinary pain providers and support staff. The same multifaceted array of stressors impacting patients has also been experienced by providers and their families. Healthcare workers face the additional burden of providing medical and mental health support to patients while managing personal stress. Data collected secondary to the severe acute respiratory syndrome (SARS) pandemic in 2003 suggests that pandemic-related stress takes a toll on the psychological wellbeing of providers [70] and is more pronounced for providers who directly treat patients with the virus [71]. Similar trends are emerging with studies conducted during the COVID-19 pandemic, where healthcare providers in Turkey [72], China [73], and the USA [74•] have reported higher rates of anxiety and depression symptoms. Although these studies are often conducted with providers directly treating patients with COVID-19, ongoing research will need to elucidate effects of the pandemic on healthcare workers across domains of practice.

\section{Conclusions}

We are only at the beginning of understanding the full ramifications of the COVID-19 pandemic on the way that children's pain is experienced and treated. Given the incidence of novel virus-related pain sequelae and increased risk across biopsychosocial factors known to initiate and exacerbate pain conditions $[8 \bullet \bullet$, there is clearly a reason to suspect an increased risk of pain among children. In the time ahead, it will be important to conduct surveillance and directed research that can provide clarity on many of the issues considered in this review and others [20••]. It will also be important to examine the ways that pain professionals and healthcare systems have demonstrated resilience by harnessing digital solutions to safely provide care for children's pain throughout this crisis. The proliferation of advancements in digital healthcare technology may provide an opportunity to transform existing paradigms of pain management, engendering a lasting impact on evidence-based pain management for children.

\section{Declarations}

Conflict of Interest Patricia A. Richardson declares that she has no conflict of interest. Anjana Kundu is founder of Proactive Pain Solutions and host of Pedia Pain Focus podcast, both of which are listed as resources within Table 1 
Human and Animal Rights and Informed Consent This article does not contain any studies with human or animal subjects performed by any of the authors.

\section{References}

Papers of particular interest, published recently, have been highlighted as:

- Of importance

-• Of major importance

1. Song XJ, Xiong DL, Wang ZY, et al. Pain management during the COVID-19 pandemic in China: lessons learned. Pain Med. 2020;21(7):1319-13230 This paper is an early report on how delivery of pain care was impacted in China in the early stages of the pandemic and recommendations for ensuring safety of the patients and healthcare professionals while providing the needed care.

2. Toubiana J, Poirault C, Corsia A, Bajolle F, Fourgeaud J, et al Kawasaki-like multisystem inflammatory syndrome in children during the covid-19 pandemic in Paris, France: prospective observational study. BMJ. 2020;369:m2094.

3. Karos K, McParland JL, Bunzli S, Devan H, Hirsh A, et al. The social threats of COVID-19 for people with chronic pain. Pain. 2020;161(10):2229-35 This article examines how the changes in basic constructs for how people live, work, interact, and access basic or healthcare needs may represent significant threats to health and wellbeing.

4. Huang Y, Zhao N. Generalized anxiety disorder, depressive symptoms and sleep quality during COVID-19 outbreak in China: a webbased cross-sectional survey. Psychiatry research. 2020;288: 112954.

5. Maugeri G, Castrogiovanni P, Battaglia G, Pippi R, D'Agata V, Palma A, et al. The impact of physical activity on psychological health during Covid-19 pandemic in Italy. Heliyon. 2020;6(6): e04315.

6. Beede D, Neville A. Broadband availability beyond the rural/urban divide. Available at: https://www.ntia.doc.gov/files/ntia/ publications/broadband_availability_rural_urban_june_2011 final.pdf. Accessed February14, 2021.

7. Reardon M. Why rural areas can't catch a break on speedy broadband. CNET, 2018. Available at: https://www.cnet.com/news/whyrural-areas-cant-catch-a-break-on-speedy-broadband/. Accessed February 14, 2021.

8.• Clauw DJ, Häuser W, Cohen SP, Fitzcharles MA. Considering the potential for an increase in chronic pain after the COVID-19 pandemic. Pain. 2020;161(8):1694-7 This article highlights the specific vulnerabilities faced by those with chronic pain during the COVID-19 pandemic and its implications for both patients and healthcare professionals in managing these patients.

9. Lacasse A, Pagé MG, Dassieu L, Sourial N, Janelle-Montcalm A, et.al. Impact of the COVID-19 pandemic on the pharmacological, physical, and psychological treatments of pain: findings from the Chronic Pain \& COVID-19 Pan-Canadian Study. Pain reports. 2021; 6(1):e891

10. Williams AC, Craig KD. Updating the definition of pain. Pain. 2016;157:2420-3

11. Palermo TM. Cognitive-behavioral therapy for chronic pain in children and adolescents. Oxford University Press; 2012;4.

12. Melzack R, Wall PD. Pain mechanisms: a new theory. Science. 1965;150(3699):971-9.
13. Gatchel RJ, Peng YB, Peters ML, Fuchs PN, Turk DC. The biopsychosocial approach to chronic pain: scientific advances and future directions. Psychological bulletin. 2007;133(4):581-624.

14. Committee on Psychosocial Aspects of Child and Family Health. The assessment and management of acute pain in infants, children, and adolescents. Pediatrics. 2001;108(3):793-7.

15. King S, Chambers CT, Huguet A, MacNevin RC, McGrath PJ, et al. The epidemiology of chronic pain in children and adolescents revisited: a systematic review. Pain. 2011;152(12):2729-38.

16. Palermo TM. Impact of recurrent and chronic pain on child and family daily functioning: a critical review of the literature. Journal of Developmental and Behavioral Pediatrics. 2000;21(1):58-69.

17. Forgeron PA, King S, Stinson JN, McGrath PJ, MacDonald AJ, Chambers CT. Social functioning and peer relationships in children and adolescents with chronic pain: a systematic review. Pain Research and Management. 2010;15(1):27-41.

18. Eccleston C, Crombez G, Scotford A, Clinch J, Connell H. Adolescent chronic pain: Patterns and predictors of emotional distress in adolescents with chronic pain and their parents. Pain. 2004;108:221-9.

19. Harrison LE, Pate JW, Richardson PA, Ickmans K, Wicksell RK, Simons LE. Best-evidence for the rehabilitation of chronic pain part 1: Pediatric pain. Journal of clinical medicine. 2019;8(9):1267.

20.• Eccleston C, Blyth FM, Dear BF, Fisher EA, Keefe FJ, et al. Managing patients with chronic pain during the COVID-19 outbreak: considerations for the rapid introduction of remotely supported (eHealth) pain management services. Pain. 2020;161(5): 889 This article examines the impact of Covid-19 pandemic on pain care delivery, and makes recommendations for rapid adaptation of alternative methods for healthcare delivery during the pandemic, based on available evidence.

21. Zou H, Lu J, Liu J, Wong JHY, Cheng S, et al. Characteristics of pediatric multi-system inflammatory syndrome (PMIS) associated with COVID-19: a meta-analysis and insights into pathogenesis. Int J Infect Dis. 2021;102:319-26.

22. Rowley AH. Understanding SARS-CoV-2-related multisystem inflammatory syndrome in children. Nat Rev Immunol. 2020;20(8): 453-4.

23.• Ashktorab Y, Brim A, Pizuorno A, Gayam V, Nikdel S, Brim H. COVID-19 pediatric patients: gastrointestinal symptoms, presentations, and disparities by race/ethnicity in a large, multi-center United States study. Gastroenterology. 2021. https://doi.org/10. 1053/j.gastro.2020.12.078 An important article providing a brief overview of the multisystem inflammatory syndrome in children and highlights the disproportionately high number of cases among the Hispanics and African American children.

24. Miller J, Cantor A, Zachariah P, Ahn D, Martinez M, Margolis K. Gastrointestinal symptoms as a major presentation component of a novel multisystem inflammatory syndrome in children (MIS-C) that is related to COVID-19: a single center experience of 44 cases. Gastroenterology. 2020;159:1571-1574.e2. https://doi.org/10. 1053/j.gastro.2020.05.079.

25. Tullie L, Ford K, Bisharat M, et al. Gastrointestinal features in children with COVID-19: an observation of varied presentation in eight children. Lancet Child Adolesc Health. 2020;4:e19-20. https://doi.org/10.1016/S2352-4642(20)30165-6.

26. Campbell A, Rodin R, Kropp R, Mao Y, Hong Z, Vachon J, et al. Risk of severe outcomes among patients admitted to hospital with pandemic (H1N1) influenza. CMAJ. 2010;182:349-55.

27. Hui DS, Chan MC, Wu AK, Ng PC. Severe acute respiratory syndrome (SARS): epidemiology and clinical features. Postgrad Med J. 2004;80:373-81.

28. Moldofsky H, Patcai J. Chronic widespread musculoskeletal pain, fatigue, depression and disordered sleep in chronic post-SARS syndrome; a case-controlled study. BMC Neurol. 2011;11:37. 
29. Hickie I, Davenport T, Wakefield D, Vollmer-Conna U, Cameron $\mathrm{B}$, Vernon SD, et al. Post-infective and chronic fatigue syndromes precipitated by viral and non-viral pathogens: prospective cohort study. BMJ. 2006;333:575.

30. Halvorson HA, Schlett CD, Riddle MS. Postinfectious irritable bowel syndrome-a meta-analysis. AmJGastroenterol. 2006;101: 1894-9.

31. Korosec-Jagodic H, Jagodic K, Podbregar M. Long-term outcome and quality of life of patients treated in surgical intensive care: a comparison between sepsis and trauma. Crit Care. 2006;10(5): R134.

32. Schelling G, Stoll C, Haller M, Briegel J, Manert W, et.al. Healthrelated quality of life and posttraumatic stress disorder in survivors of the acute respiratory distress syndrome. Crit Care Med. 1998;26: 651-659.

33. Timmers TK, Verhofstad MH, Moons KG, van Beeck EF, Leenen LP. Long-term quality of life after surgical intensive care admission. Arch Surg. 2011;146:412-8.

34. Ellis J, Sharp D, Newhook K, Cohen J. Selling comfort: a survey of interventions for needle procedures in a pediatric hospital. Pain Management Nursing. 2004;5(4):144-52.

35. Rabbitts JA, Fisher E, Rosenbloom BN, Palermo TM. Prevalence and predictors of chronic postsurgical pain in children: a systematic review and meta-analysis. The journal of pain. 2017;18(6):605-14.

36. Campo JV, Comer DM, Jansen-McWilliams L, Gardner W, Kelleher KJ. Recurrent pain, emotional distress, and health service use in childhood. Journal of Pediatrics. 2002;141:76-83.

37. Cunningham NR, Cohen MB, Farrell MK, Mezoff AG, LynchJordan A, Kashikar-Zuck S. Concordant parent-child reports of anxiety predict impairment in youth with functional abdominal pain. J Pediatr Gastroenterol Nutr. 2015;60(3):312-7.

38. Cunningham NR, Tran ST, Lynch-Jordan AM, Ting TV, Sil S, Strotman D, et al. Psychiatric disorders in young adults diagnosed with juvenile fibromyalgia in adolescence. J Rheumatol. 2015;42(12):2427-33.

39. Larsson B, Sund AM. Emotional/behavioural, social correlates and one-year predictors of frequent pains among early adolescents: influences of pain characteristics. Eur J Pain. 2007;11(1):57-65.

40. Racine N, Cooke JL, Eirich R, Korczak DJ, McArthur B, Madigan S. Child and mental illness during COVID-19: a rapid review. Psychiatry research. 2020;292:113307.

41. YoungMinds. (2020). Coronavirus: Impact on young people with mental health needs. https://youngminds.org.uk/media/3708/ coronavirus-report march2020.pdf. Accessed March 1, 2021.

42. Groenewald CB, Tham SW, Palermo TM. Impaired school functioning in children with chronic pain: a national perspective. The Clinical Journal of Pain. 2020;36(9):693-9.

43. Logan DE, Simons LE, Stein MJ, Chastain L. School impairment in adolescents with chronic pain. The Journal of Pain. 2008;9(5):40716.

44. Fales JL, Forgeron PA. Commentary: The importance of friendships in youth with chronic pain: the next critical wave of research. Pediatric Pain Letter. 2016;16(3):35-9.

45. Kuhfield M, Soland J, Tarasawa B, Johnson A, et al. Projecting the potential impacts of COVID-19 school closures on academic achievement. EdWorkingPaper Available: (2020), pp. 20-226 https://edworkingpapers.com/sites/default/files/ai20-226-v2.pdf. Accessed March 1, 2021.

46. United States Department of Agriculture Food security in the United States. https://www.ers.usda.gov/data-products/foodsecurity-in-the-united-states/ Date accessed: March 25, 2020

47. Kichline T, Cushing CC, Ortega A, Friesen C, Schurman JV. Associations between physical activity and chronic pain severity in youth with chronic abdominal pain. The Clinical journal of pain. 2019;35(7):618-24.
48. Clinch J, Eccleston C. Chronic musculoskeletal pain in children: assessment and management. Rheumatology (Oxford). 2009;48: 466-74.

49. Fallon N, Brown C, Twiddy H, Brian E, Frank B, et.al. Adverse effects of COVID-19-related lockdown on pain, physical activity and psychological well-being in people with chronic pain. British Journal of Pain 2020; 2049463720973703.

50. Valrie CR, Bromberg MH, Palermo TM, Schanberg LE. A systematic review of sleep in pediatric pain populations. Journal of developmental and behavioral pediatrics. 2013;34(2):120-8. https://doi. org/10.1097/DBP.0b013e31827d5848.

51. Cusinato M, Iannattone M, Spoto A, Poli M, Moretti M, et al. Stress, Resilience, and Well-Being in Italian Children and Their Parents during the COVID-19 Pandemic. Int J Environ Res Public Health. 2020;17(22):8297. https://doi.org/10.3390/ ijerph17228297.

52. Groenewald CB, Murray CB, Palermo TM. Adverse childhood experiences and chronic pain among children and adolescents in the United States. Pain Reports. 2020;5(5):e839 This article discussed the correlation between the Adverse Childhood Events and chronic pain, therefore implications to closely track the incidence and prevalence of the post pandemic pain.

53. Sachs-Ericsson NJ, Sheffler JL, Stanley IH, Piazz JR, Preacher KJ. When emotional pain becomes physical: adverse childhood experiences, pain, and the role of mood and anxiety disorders. Journal of clinical psychology. 2017;73(10):1403-28.

54. Häuser W, Kosseva M, Üceyler N, Klose P, Sommer C. Emotional, physical, and sexual abuse in fibromyalgia syndrome: a systematic review with meta-analysis. Arthritis Care Res. 2011;636:808-20.

55. You DS, Albu S, Lisenbardt H, Meagher MW, et al. Pain Medicine. 2019;20(3):486-94 This article highlights the correlation between adverse factors in childhood that are likely to be magnified during the pandemic and the likelihood of occurrence of pain.

56. CDC injury prevention and control.

57. Baron EJ, Goldstein EG, Wallace CT. Suffering in silence: How COVID-19 school closures inhibit the reporting of child maltreatment. Journal of public economics. 2020;190:104258 This article highlights the disadvantaged position of children with compromised safety due to the school closures related to the pandemic, and lack of ability to identify or report such events, which in turn may result in increased pain complaints along with the abuse.

58. Jansen M, Irving H, Gillam L, Sharwood E, Preisz A, Basu S, et al. Ethical considerations for paediatrics during the COVID-19 pandemic: a discussion paper from the Australian Paediatric Clinical Ethics Collaboration. J Paediatr Child Health. 2020;56(6):847-51.

59. Thornton J. During the past few months, a significant decline in general practitioner appointments, specialized care, and pediatric emergency department attendance. BMJ. 2020;369:m1401.

60. Schechter NL, Berde CB, Yaster M. Pain in infants, children, and adolescents: an overview. In: Schechter NL, Berde CB, Yaster M, editors. Pain in Infants, Children, and Adolescents. Baltimore, MD: Williams \& Wilkins; 1993. p. 3-9.

61. Palermo TM, Slack M, Zhou C, Aaron R, Fisher E, Rodriguez S. Waiting for a pediatric chronic pain clinic evaluation: a prospective study characterizing waiting times and symptom trajectories. The Journal of Pain. 2019;20(3):339-47.

62. Lynch ME, Campbell F, Clark AJ, Dunbar MJ, Goldstein D, Peng $\mathrm{P}$, et al. A systematic review of the effect of waiting for treatment for chronic pain. Pain. 2008;136(1):97-116. https://doi.org/10. 1016/j.pain.2007.06.018.6.

63. Peng $\mathrm{P}$, Choiniere $\mathrm{M}$, Dion $\mathrm{D}$, Intrater $\mathrm{H}$, Lefort $\mathrm{S}$, Lynch $\mathrm{M}$, et al. Challenges in accessing multidisciplinary pain treatment facilities in Canada. Can J Anaesth. 2007;54:977-84. https://doi.org/10. 1007/BF03016631. 
64. Kuo DZ, Cohen E, Agrawal R, Berry JG, Casey PH. A national profile of caregiver challenges among more medically complex children with special health care needs. Archives of pediatrics \& adolescent medicine. 2011;165(11):1020-6.

65. Sannicandro T, Parish SL, Son E, Powell RM. Health care changes for children with special health care needs, 2005-2011. Maternal and child health journal. 2017;21(3):524-30.

66. Palermo T. Remote management of pediatric pain. Encyclopedia of Pain. 2nd ed. New York: Springer; 2013. p. 3389-93.

67. Tauben DJ, Langford DJ, Sturgeon JA, Rundell SD, Towle C, et al. Optimizing telehealth pain care after COVID-19. Pain. 161(11): 2437-45 This article takes a close look at the benefits and pitfalls of rapid adaptation of telehealth for pain management and recommends future areas for improvement.

68. Stephenson J. https://jamanetwork.com/channels/health-forum/ fullarticle $/ 2772241$ ? utm_source=fbpage\&utm_medium $=$ social jamaforum\&utm_term $=3954797460 \& u t m \_$campaign $=$article alert\&linkId $=102447361$

69. Boyd CJ, McCabe SE, Cranford JA, Young A. Adolescents' motivations to abuse prescription medications. Pediatrics. 2006;118(6): 2472-80. https://doi.org/10.1542/peds.2006-1644 This paper highlights the factors that contribute to medication abuse /misuse and the implications it may have on pain management of youth during the pandemic as well as a need for surveillance of such factors.
70. Bai Y, Lin CC, Lin CY, Chen JY, Chue CM, Chou P. Survey of stress reactions among health care workers involved with the SARS outbreak. Psychiatric Services. 2004;55(9):1055-7.

71. Maunder RG, Lancee WJ, Balderson KE, Bennett JP, Borgundvaag B, Evans S, et al. Long-term psychological and occupational effects of providing hospital healthcare during SARS outbreak. Emerg Infect Dis. 2006;12:1924-32.

72. Elbay RY, Kurtulmuş A, Arpacioğlu S, Karadere E. Depression, anxiety, stress levels of physicians and associated factors in Covid19 pandemics. Psychiatry research. 2020;290:113130.

73. Chen Y, Zhou H, Zhou Y, Zhou F. Prevalence of self-reported depression and anxiety among pediatric medical staff members during the COVID-19 outbreak in Guiyang. China. Psychiatry research. 2020;288:113005.

74. Shechter A, Diaz F, Moise N, Anstey DE, Ye S, Agarwal S, et.al. (2020). Psychological distress, coping behaviors, and preferences for support among New York healthcare workers during the COVID-19 pandemic. General hospital psychiatry. 2020;66:1-8. This article highlights the toll COVID-19 pandemic has had on healthcare professionals well-being and the need for an escalation in support

Publisher's Note Springer Nature remains neutral with regard to jurisdictional claims in published maps and institutional affiliations. 